\title{
A Robust Video Watermarking Algorithm Based on Spatial Domain
}

\author{
Guangxi Chen", a , Chunsheng Kang, 2, b, Dao shun Wang', c, \\ Xianghui Zhao ${ }^{3, d}$ and Yong Huang ${ }^{4, e}$
}

${ }^{1}$ School of Computer Science and Information Security, Guilin university of electronic science and technology, Guilin 541000, China

${ }^{2}$ School of computing, Tsinghua University, Beijing 100000, China

${ }^{3}$ China Information Technology Security Evaluation Center, Beijing 100000, China

${ }^{4}$ School of Computer Science and Education Software, Guangzhou University, Guangzhou 510000, China

achgx@guet.edu.cn, b1176154395@qq.com, ${ }^{\mathrm{c}}$ daoshun@mail.tsinghua.edu.cn, dzhaoxianghui@mail.itsex.gov.cn, ${ }^{\mathrm{e}} 79031404 @ q q . c o m$

\begin{abstract}
Keywords: Video watermarking, High Robustness, Spatial Domain, Visual Quality
Abstract. Compared with the transform domain video watermarking algorithms, the spatial watermarking algorithms have weak robustness, but they have high visual quality. To ensure the video quality and satisfy the robustness requirement, a robust video watermarking algorithm based on spatial domain is proposed. Firstly, the video sequence is divided into five frames as a group. Secondly, We use luminance adaptive and edge mask to select the embedding area and determine the embedding strength adaptively. Then, the watermark information is embedded by fine-tuning the luminance trend of five consecutive frames. Finally, watermark information is extracted by the overall luminance of video sequences. Experimental results show that compared with the transform domain algorithms, the proposed algorithm makes a balance between robustness and visual quality, it improves the video quality and meets the robustness requirement under scaling, compression and recoding.
\end{abstract}

\section{Introduction}

Video watermarking technology is widely used in video copyright protection, video authentication and public surveillance, and the watermark information can be distributed with the distribution of videos. However, the network propagation of videos is easily affected under scaling, compression and recoding. Therefore, the robust video watermarking algorithm is used to ensure the integrity of watermark information during video propagation.

There exist many video watermarking algorithms based on transform domain. Buhari et al. [1] proposed a fast watermarking scheme which could resist the attacks of recoding and Gauss filtering, but it reduces visual quality. Fung et al. [2] proposed the video watermarking algorithm to improve the robustness and accuracy of watermark extraction, but it may reduce visual quality. Wang et al. [3] used corners and singular value decomposition to embed the watermarking information, it has a high robustness under transcoding, noise and recoding. What's more, it improves the detection rate of the watermark and reduces the block effect and flicker. Li et al. [4] proposed a watermarking scheme, which has good visual quality and robust to attacks under compression, transcoding, recoding and geometric transformation. However, it exists block effects on the smooth regions. Zhang et al. [5] proposed a novel data hiding method, it has a high robustness against compression and quantization attacks, but it reduces video quality. Gaj et al. [6] proposed a robust video watermarking algorithm, it has a high robustness to compression and recoding attacks, but it reduces visual quality when the watermark capacity increases.

Simultaneous the spatial domain watermarking algorithms are as follows. Mohammed et al. [7] proposed a robust video watermarking algorithm. It improves the video quality and has well robustness for geometric attacks, recoding, transcoding and other attacks. Authors in refs. [8,9] 
proposed algorithms were based on using the pixel histogram. They have a high visual quality and can resist the attacks of compression and transcoding, but its robustness is weak for re-coding and geometric attacks. Haitsma et al. [10] changed the overall brightness to embed the watermark into each frames, but its robustness is weak.

The analysis and experiments indicate that the transform domain video watermarking algorithms have a high robustness, but they have high complexity and may reduce the video quality. Although the robustness of spatial domain watermarking algorithms is weak, the video quality is high. To ensure video quality and improve the robustness of spatial domain watermarking algorithms, we propose a robust video watermarking algorithm based on spatial domain.

This paper is organized as follows. In section 2, we analyze the spatial domain JND model and the watermarking algorithm based on time modulation. In section 3, we describe the embedding process and extraction process of the proposed algorithm. In section 4, we compare and analyze the proposed algorithm video quality and robustness. In section 5, we give a conclusion.

\section{Background and preliminaries}

Spatial domain JND model. Many video watermarking algorithms based on the spatial domain JND model can get the embedded region and watermark intensity by using luminance adaptation, contrast mask and contrast sensitivity function. They can achieve high visual quality and have a high robustness to the attacks under compression, recoding, transcoding and so on. For example, Qi et al. [11] used the brightness adaptive and texture masking to ensure the watermark intensity. Authors in refs. $[12,13,14]$ improved the JND (minimum perceptible difference) model. The embedding area and watermark intensity are determined by the relationship between the luminance mask, the contrast mask and the temporal-spatial fusion mask. Our paper introduces the luminance adaptive and contrast mask.

(1) Luminance Adaptation(LA). Because the sensitivity of HVS to luminance difference depends on the local intensity of the image region, it is less sensitive to the noise of the darker and brighter regions. What's more, Guo et al. [13] proposed that the increase of background brightness could increase the minimum perceptible luminance difference. The luminance adaptive mask $L A(x, y)$ is calculated as follows.

$$
L A(x, y)= \begin{cases}17 \times(1-\sqrt{f(x, y) / 127})+3 & f(x, y) \leq 127 \\ 3 \times(f(x, y)-127) / 128+3 & \text { otherwise }\end{cases}
$$

$f(x, y)$ represents the pixel value of the image at the point $(x, y)$, the range of $\mathrm{x}$ and $\mathrm{y}$ are $0 \leq x<m, 0 \leq y<n$, $\mathrm{m}$ and $\mathrm{n}$ are the length and width of the images, respectively.

(2) Contrast Mask(CM). Generally, the contrast mask is mainly determined by the texture and edge of images. Therefore, the calculation formula of the contrast mask (CM) is as follows:

$$
\begin{aligned}
C M(x, y) & =E M^{m}(x, y)+T M^{n}(x, y) \\
E M^{m}(x, y) & =C(x, y) \times p \\
T M^{n}(x, y) & =C(x, y) \times q
\end{aligned}
$$

$E M^{m}$ and $T M^{n}$ represent the edge and texture regions respectively, $\mathrm{p}$ and $\mathrm{q}$ are used to distinguish edge information from the texture mask, set to 1 and 3 respectively. $C(x, y)$ represents the spatial contrast at the point $(x, y)$.

Therefore, the value of JND can be expressed as:

$$
J N D(x, y)=L A(x, y)+C M(x, y)-\beta(\min (L A(x, y), C M(x, y)))
$$

Compared with the texture region, the edge structure is simpler and more predictable, and the existing JND model does not accurately distinguish the edge and texture regions. And HVS has less 
sensitive to the change of the edges. Image edge areas have a high resistance to the existing compression standards (such as H.264, MPEG4, etc.), and it can keep high video quality [12,15]. So our paper adopts edge mask and luminance adaptive mask to generate watermark template.

Video watermarking algorithms based on time modulation. The time modulation mainly adjusts the luminance of the frame groups based on time axis to embed the watermark. Refs. [4] used the improved waston model for getting the embedding strength, fine-tuning the luminance relationship of the frame groups to embed the watermark. The embedding process as follows.

Input: Original video $V$, the watermark information $w$

Output: Embedded video $V^{\prime}$

Step1: The original video $V$ is divided into one frame group per $\mathrm{K}$ frame, and obtains the luminance component of each frame.

Step2: Each frame is divided into the non-overlapping $32 \times 32$ macro-block, then divide each block into 16 non-overlapping $8 \times 8$ blocks, calculate the DC coefficient of $8 \times 8$ blocks, and calculate the average DC coefficient of each macro-block according to the DC coefficient of each block.

Step3: According to the watson brightness masking model calculates the value of JND in $8 \times 8$ block DCT domain. Then, analysis the relationship between the JND value and the average luminance value of the spatial domain, the average value of the luminance components in the spatial is adjusted to get the appropriate embedded watermark intensity.

Step4: Modifying the watermark intensity and the value of $4 \times 4$ blocks with the maximum brightness of $8 \times 8$ blocks to obtain the watermark template $M$.

Step5: If the watermark bit is one, the first $\mathrm{K} / 2$ (the integral part) frame of each frame group was added with $\mathrm{M}$ and the latter $\mathrm{K} / 2$ frame minus $M$.

If the watermark bit is zero, the first $\mathrm{K} / 2$ frames of each frame group were subtracted from $M$, followed by $\mathrm{K} / 2$ frames plus $M$.

Step6: Repeat the above steps until the watermark information is embedded, and get the watermarked video.

Through the analysis of the embedding algorithm, the original video sequences are divided into a group of $\mathrm{K}$ frames, then the luminance components of each frame groups are divided into $32 \times 32$ blocks which are not overlapping, and then divided into $8 \times 8$ blocks, and by calculating the DC coefficients of each $8 \times 8$ blocks to obtain the average DC coefficients of $32 \times 32$ blocks.

First, according to the improved waston model ensure the embedded watermark intensity in order to obtain the watermark template. Then adjusting the block with the maximum average luminance in each $8 \times 8$ block. Through calculating, there are four kinds of watermark patterns in $8 \times 8$ blocks:

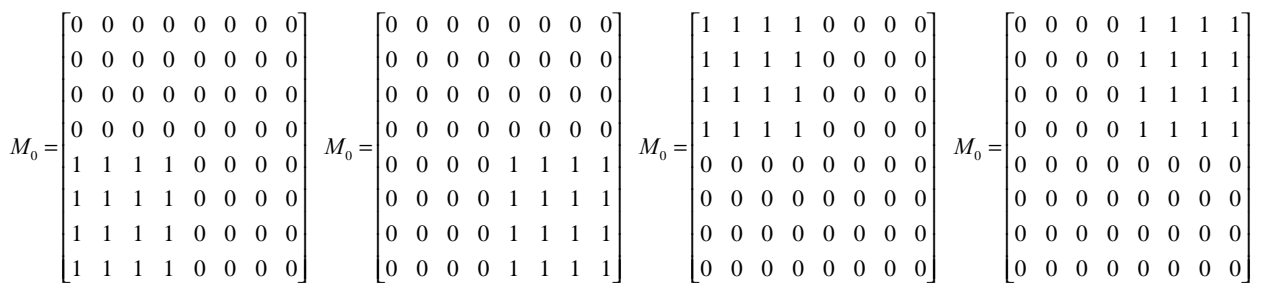

According to the distribution of the watermark template value, it can be seen that the embedding of the watermark is the whole modification of the $4 \times 4$ block in the $8 \times 8$ block of the luminance component, which will lead to the discontinuity of the boundary between the adjacent blocks. The situation that some block regions will be brightened or darkened in the image, thus bringing block effect. The experiment shows that the algorithm will have a significant block effect on large area pure color frames, affecting visual perception, as shown in Fig. 1. 



Fig. 1 Block effect schematic diagram

\section{The Proposed Algorithm}

Embedding algorithm. The embedding is mainly based on the spatial JND model, using the luminance adaptive and improved edge mask to generate the watermark template, so that adopting adaptive the embedding intensity and region. The improved edge mask (EM) is generated by the relationship between edge image and luminance value. So the calculation formula of the edge mask factor is as follows:

$$
E M(x, y)=\gamma \times \frac{E(x, y)}{E_{\max }} \quad 0 \leq x<m, 0 \leq y<n
$$

$E(x, y)$ represents the luminance value of edge image at the point $(x, y), E_{\max }(x, y)$ is the maximum luminance of the edge image. $\gamma$ is the adjustment coefficient. Moreover, $m$ and $n$ are the image's length and width, respectively. Therefore, the value of watermark template $\mathbf{M}$ at point $(x, y)$ is:

$$
M(x, y)=L A(x, y)+E M(x, y)-\beta(\min (L A(x, y), E M(x, y))) \quad 0 \leq x<m, 0 \leq y<n
$$

So, if $F^{k}$ is the luminance components of the original video sequence in the $k$ frame, $F_{w}^{k}$ is the luminance components of the embedded video sequence in the $k$ frame. $M^{k}$ is the watermark template in the $k$ frame. The particular embedding formula is:

$$
F_{w}^{k}(x, y)=F^{k}(x, y) \pm \lambda g M^{k}(x, y) \quad 0 \leq x<m, 0 \leq y<n
$$

$F^{k}(x, y)$ is the luminance value of $F^{k}$ at point $(x, y) . F_{w}^{k}(x, y)$ is the luminance value of $F_{w}^{k}$ at point $(x, y)$. $M^{k}(x, y)$ is the value of $M^{k}$ at point $(x, y)$, The range of $k$ is $0<k \leq N, N$ is the number of frames for video. $\mathrm{g}$ is the adjustment coefficient, $\lambda$ is used to adjust the watermark information.. The adjustment method is:

$$
\lambda= \begin{cases}1 & w=1 \\ -1 & w=0\end{cases}
$$

The specific embedding algorithm is as follows.

\section{Input: original video $V$, watermark information $w$}

Output: the embedded video $V^{\prime}$

Step1: Convert the video frame sequences from RGB space to YUV space, then the video frames are divided into frame group(five frames), then extract the $\mathrm{Y}$ channel information of every frames.

Step2: Calculate the brightness adaptive mask LA for each frame groups through the luminance adaptive model.

Step3: Extracting the edge information, and calculating the edge mask (EM), then using the LA and EM to obtain the watermark template.

Step4: Obtaining the watermark information after processing (value is $\{0,1\}$ ). If the watermark bit is 1 , the luminance value of every groups are adjusted to:

$$
F_{w}^{k}(x, y)=\left\{\begin{array}{l}
F^{k}(x, y)+g M^{k}(x, y) \quad k \bmod 5=1,2 \\
F^{k}(x, y) \quad k \bmod 5=3 \\
F^{k}(x, y)-g M^{k}(x, y) \quad k \bmod 5=0,4
\end{array}\right.
$$

if the watermark bit is 0 , the luminance value of the frames are adjusted to:

$$
F_{w}^{k}(x, y)=\left\{\begin{array}{l}
F^{k}(x, y)-g M^{k}(x, y) \quad k \bmod 5=1,2 \\
F^{k}(x, y) \quad k \bmod 5=3 \\
F^{k}(x, y)+g M^{k}(x, y) \quad k \bmod 5=0,4
\end{array}\right.
$$

Step5: Until the watermark information is fully embedded into the video sequences. 
Extraction algorithm. The extraction process is from the extraction algorithm in refs. [4]. The method is a blind watermarking algorithm. Firstly, decode one chosen single-shot video and get the watermarked frame groups. Secondly, change the RGB color space into YUV space and obtain the Y channel. Then, take 1/4 of the area in the middle of the Y channel of consecutive frames as the detecting area. Finally, get the information based on the luminance trend in the detecting area in the consecutive five frames. Fig. 2 shows the luminance trend (the red curve represents the original video luminance trend, and the blue represents the watermarked video luminance trend).

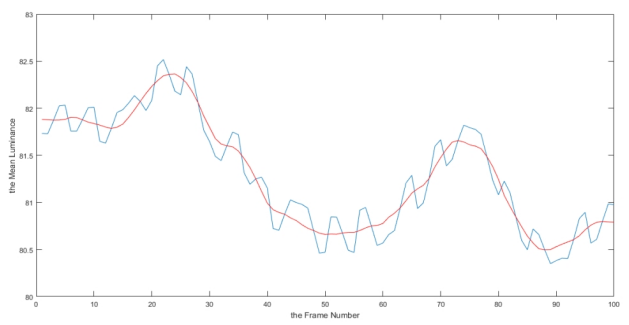

Fig. 2 The luminance trend curve

\section{Analysis of experimental results}

Block effect analysis. Randomly select consecutive frames of watermarked video to observe the watermark template value of $8 \times 8$ blocks per frame. The first frame, the second frame, the fourth frame and fifth frame in the same position of the $8 \times 8$ blocks value matrix are $M_{1}, M_{2}, M_{4}$ and $M_{5}$ respectively.

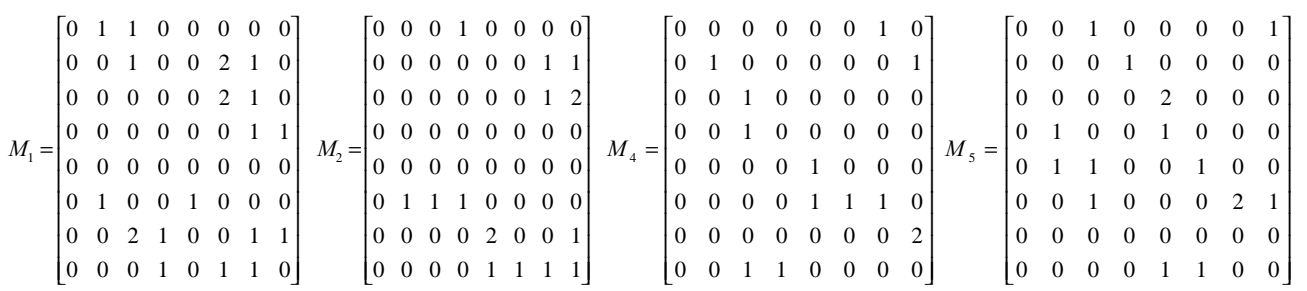

From the value of watermark template finds that the embedding area and strength of watermark are random. And the changes of luminance focus on image edge regions. Compared with the watermark template of refs. [4], our method can adaptively get the embedding region and watermark strength without causing block effect.

Test result analysis. Five standard video sequences are selected in the test experiment. Seq1, Seq2 and Seq3 are all from the video sequence for special test in the film detection institute, and the resolution is $1920 \times 1080$. Seq4 and Seq5 are standard test videos(bridge and mobile), and the resolution is $352 \times 288$.

(1) video quality analysis. Through the observation of subjective vision, the visual quality of videos has not changed significantly. Fig. 3 shows the original videos and watermarked videos.


Seq2 

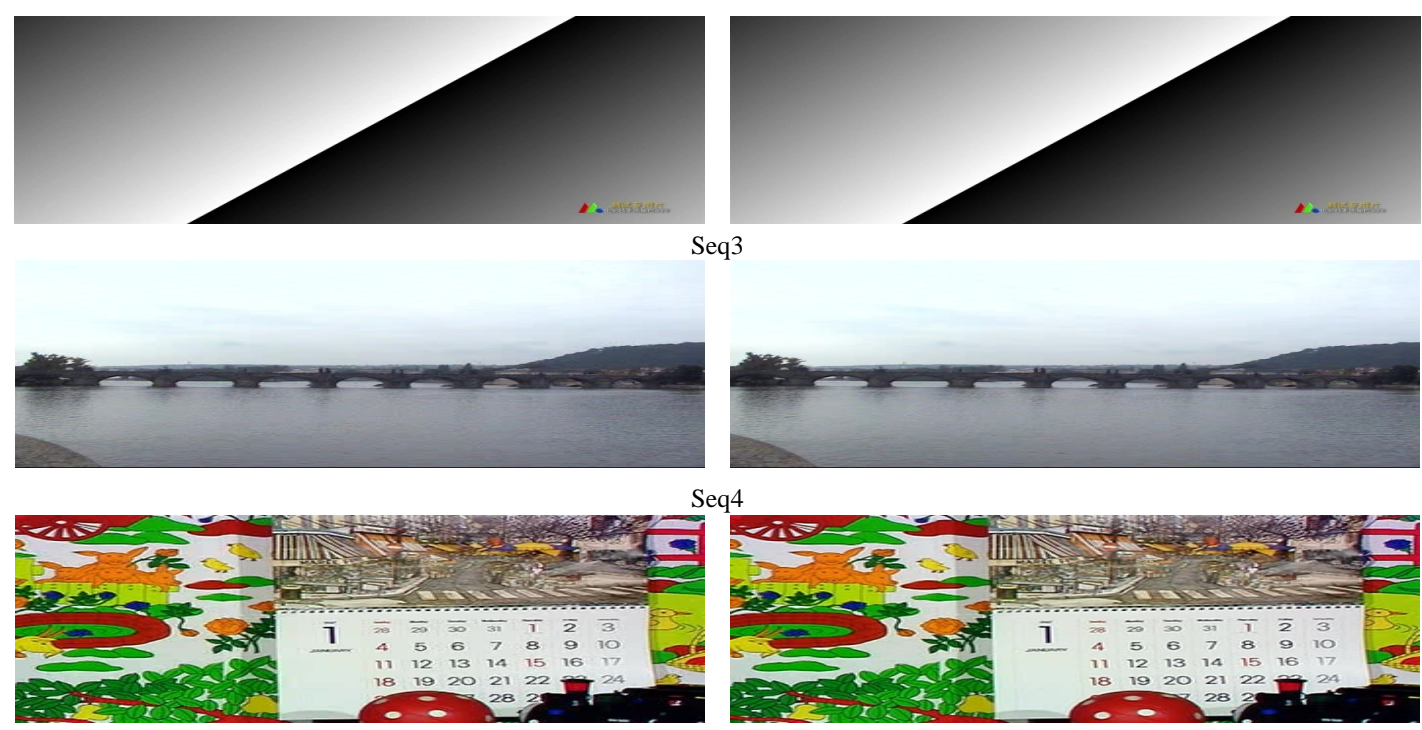

eq4

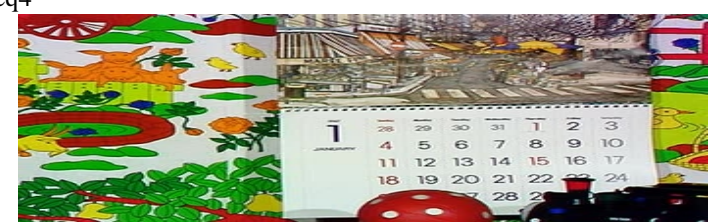

Seq5

Fig. 3 The original videos and watermarked videos

(the original videos are on the left, the watermarked videos are on the right)

This paper mainly adopts the PSNR and MSDS [17]. PSNR is used to evaluate the overall quality of videos. The higher the value, the better the quality of videos. MSDS is used to calculate the average block effect of images. The larger the value, the more serious the video block phenomenon. The PSNR values and MSDS values are shown in Table1 and Table 2 respectively.

Table1 The average PSNR of video

\begin{tabular}{|c|c|c|}
\hline Videos & paper[4] & Our method \\
\hline Seq1 & 44.05 & 55.57 \\
\hline Seq2 & 46.63 & 52.27 \\
\hline Seq3 & 48.06 & 52.43 \\
\hline Seq4 & 48.14 & 50.01 \\
\hline Seq5 & 51.89 & 54.52 \\
\hline Average & 47.75 & 52.96 \\
\hline
\end{tabular}

The experimental results in Table 1 show that the average PSNR value of our method is more than $5 \mathrm{~dB}$, compared with the paper [4]. The results in Table 2 show that the MSDS values are lower than the paper [4], except that the Seq5 contains abundant edge information. The results in Tables 1 and 2 show that the video quality is better than the paper [4].

(2) Robustness analysis. Our paper uses the format factory to test the watermark detection rate for the attacks, such as scaling, H. 264 compression and recoding. The results are shown in the Table 3.

Table 3 The watermark detection rate

\begin{tabular}{|c|c|c|c|c|c|c|}
\hline \multirow{2}{*}{ Attacks } & \multicolumn{2}{|c|}{ Scale } & \multicolumn{2}{c|}{ H.264compression } & \multicolumn{2}{c|}{ recoding } \\
\cline { 2 - 7 } & paper[4] & Our method & paper [4] & Our method & paper [4] & Our method \\
\hline Seq1 & 1 & 1 & 1 & 0.95 & 1 & 0.95 \\
\hline Seq2 & 1 & 1 & 1 & 0.93 & 1 & 0.93 \\
\hline Seq3 & 1 & 1 & 1 & 0.98 & 1 & 0.98 \\
\hline Seq4 & 1 & 1 & 1 & 0.95 & 1 & 0.93 \\
\hline Average & 1 & 1 & 1 & 0.958 & 1 & 0.953 \\
\hline
\end{tabular}

In addition, according to the analysis finds that the process of videos propagation will encounter combination attacks under compression, scaling, recoding, including scaling, compression, recoding and transcoding. Some video watermarking algorithms do not involve the analysis and test the robust for the combined attacks. Therefore, our paper uploads the embedded watermark videos to Tencent, 
Sohu, 56 video, Youku and other video websites, then extracts the watermark information from the downloaded videos. The results are shown in the Table 4.

Table4 The watermark detection accuracy of video websites

\begin{tabular}{|c|c|c|c|c|}
\hline $\begin{array}{c}\text { Website } \\
\text { Video }\end{array}$ & Tencent & Sohu & 56 video & Youku \\
\hline Seq1 & 0.95 & 0.95 & 0.925 & 0.925 \\
\hline Seq2 & 0.825 & 0.825 & 0.80 & 0.80 \\
\hline Seq3 & 0.85 & 0.867 & 0.883 & 0.817 \\
\hline Seq4 & 0.800 & 0.783 & 0.833 & $/$ \\
\hline Average & 0.856 & 0.856 & 0.860 & 0.847 \\
\hline
\end{tabular}

The experimental results in Table 4 show that the average accuracy of video extraction in the process of video propagation is close to $86 \%$, mainly due to the different compression and coding methods of different video websites decrease the accuracy rate. In addition, the watermark information of different video websites (such as Youku video, Tencent video, etc.) will be embedded again in the process of video upload, which has a great influence on the extraction of watermark information.

\section{Conclusions}

Video transmission in the network will encounter scaling, compression, recoding and other combination attacks. Compared with the transform domain watermarking algorithms, the spatial domain video watermarking algorithms can ensure the videos quality but its robustness is weak. To ensure the video quality and improve the robustness of spatial domain algorithm, our paper proposes a robust video watermarking algorithm based on spatial domain. Using luminance adaptation and edge mask to selects embedded region and watermark intensity adaptively. Then tuning the luminance relationship of the frame groups to embed the watermark information. Finally, according to the overall luminance trend of the video frames extracts the watermark. Experimental results show that compared with the transform domain watermarking algorithms, the proposed algorithm slightly improves the video quality and meets the robustness requirement. Simultaneously, the feasibility and robustness of the algorithm are verified by the experiments on different video websites.

\section{Acknowledgements}

This work was supported in part by the National Natural Science Foundation of China under Grant 61373020, Grant U1536102, and Grant U1536116, in part by China Mobile Research Fund Project (MCM20170407), the National Natural Science Foundation of China under Grant Nos. 61462018 and Guangzhou University mathematics Education Software Research Center Open Foundation under Grant Nos. LD16124X.

\section{References}

[1] [1] A.M. Buhari, H.C. Ling, V.M. Baskaran, et al. Fast watermarking scheme for real-time spatial scalable video coding[J]. Signal Processing Image Communication, 2016, 47(C):86-95.

[2] C.W. Fung, W.G. Jr. A New Approach of DWT-SVD Video Watermarking[C]// Third International Conference on Computational Intelligence, Modelling \& Simulation. IEEE Computer Society, 2011:233-236.

[3] D.S Wang, S. Liu, X.Y. Luo. A transcoding-resistant video watermarking algorithm based on corners and singular value decomposition[J]. Telecommunication Systems, 2013, 54(3):359-371.

[4] L. Li, Z. Dong, J. Lu, et al. An H.264/AVC HDTV watermarking algorithm robust to camcorder 
recording[J]. Journal of Visual Communication \& Image Representation, 2015, 26(C):1-8.

[5] H. Zhang, Y. Cao, X. Zhao, et al. Data Hiding in H.264/AVC Video Files Using the Coded Block Pattern[C]// International Workshop on Digital Watermarking. Springer International Publishing, 2016:588-600.

[6] S. Gaj, A. Sur, P. K. Bora. A robust watermarking scheme against re-compression attack for H.265/HEVC[C]// Computer Vision, Pattern Recognition, Image Processing and Graphics. IEEE, 2016:1-4.

[7] A. A. Mohammed, N. A. Ali. Robust video watermarking scheme using high efficiency video coding attack[J]. Multimedia Tools \& Applications, 2017(3):1-16.

[8] H. Do, D. Choi, T. Kim, et al. Digital video watermarking based on histogram and temporal modulation and robust to camcorder recording[C]// IEEE International Symposium on Signal Processing and Information Technology. IEEE, 2008:330-335.

[9] X.F. Hu, D. S. Wang. A Histogram Based Watermarking Algorithm Robust to Geometric Distortions[C]// International Conference on Electrical, Computer Engineering and Electronics. 2015.

[10] J. Haitsma, T. Kalker. A watermarking scheme for digital cinema[C]// International Conference on Image Processing, 2001. Proceedings. IEEE Xplore, 2001:487-489.

[11]H. Qi, J. Zhao. HVS based adaptive digital image watermarking[C]// Electrical and Computer Engineering, 2006. CCECE '06. Canadian Conference on. IEEE, 2007:302-305.

[12] M. Uzair, R.D. Dony. Estimating just-noticeable distortion for images/videos in pixel domain[J]. Iet Image Processing, 2017, 11(8):559-567.

[13] J. Guo, Y. Zhang, S. Zhi, et al. Adaptive edge masking based on TV decomposition and adjacent similarity for digital watermarking[C]// IEEE International Conference on Progress in Informatics and Computing. IEEE, 2016:142-147.

[14] W. Zhang, X. Li, Y. Zhang, et al. Robust video watermarking algorithm for H.264/AVC based on JND model[J]. Ksii Transactions on Internet \& Information Systems, 2017, 11(5):2741-2761.

[15]P.R. Kumar, K.L. Sailaja. Watermarking algorithm using sobel edge detection[J]. International Journal of Advanced Networking \& Applications, 2011, 2(5).

[16] A. Liu, W. Lin, M. Paul, et al. Just noticeable difference for images with decomposition model for separating edge and textured regions[J]. IEEE Transactions on Circuits \& Systems for Video Technology, 2010, 20(11):1648-1652.

[17] G. A. Triantafyllidis, D. Tzovaras, M. G. Strintzis. Reduction of blocking artifacts in block-based compressed images[J]. 2005, 3708:419-426. 